\title{
Inclusion of Bandstructure and Many-Body Effects in a Quantum Well Laser Simulator
}

\author{
F. OYAFUSO*, P. von ALLMEN, M. GRUPEN and K. HESS \\ Beckman Institute, University of Illinois, Urbana, IL 61801
}

\begin{abstract}
A self-consistent eight band $\mathbf{k} \cdot \mathbf{p}$ calculation, which takes into account strain and includes Hartree, exchange, and correlation terms (determined from a local density approximation) is incorporated into a QW laser simulator (MINILASE-II). The computation is performed within the envelope function approximation for a superlattice, in which all spatially varying terms of the k.p Hamiltonian, including the exchange and correlation energies are expanded in plane waves. The $\mathbf{k} \cdot \mathbf{p}$ eigenvalue equation, and Poisson's equation are solved iteratively until self-consistency is attained. Results from the $\mathbf{k} \cdot \mathbf{p}$ calculation are exported to MINILASE-II via a density of states and an energy dependent optical matrix element factor, renormalized by a Coulomb enhancement factor to account for electron-hole attraction. Results are presented for the gain spectrum and modulation response for a $\mathrm{Ga}_{0.8} \mathrm{In}_{0.2} \mathrm{As} / \mathrm{Al}_{0.1} \mathrm{Ga}_{0.9}$ As quantum well laser with and without the inclusion of the Coulomb enhancement factor.
\end{abstract}

Keywords: Gain, k·p, laser, quantum well, modulation response, bandstructure

MINILASE-II [1] is a semiconductor laser simulator that self-consistently solves Poisson's equation, the carrier transport equations (both drift diffusion and thermionic emission across heterostructures), quantum well (QW) capture, and the photon rate equations for arbitrary two dimensional geometries. Many important effects arise from the bandstructure near the quantum well. However, it is computationally impractical to perform an accurate self-consistent bandstructure calculation within the laser simulator at each iteration. The purpose of this paper is to explain how we connect a separate eight band $\mathbf{k} \cdot \mathbf{p}$ superlattice calculation, including many-body effects, with MINILASE-II and to present results for the gain and modulation response for a strained-layer $\operatorname{In}_{0.2} \mathrm{Ga}_{0.8} \mathrm{As} / \mathrm{Al}_{0.1} \mathrm{Ga}_{0.9}$ As quantum well laser.

The k.p calculation involves diagonalizing a Hamiltonian which at the center of the Brillouin zone can be expressed as

$$
\begin{aligned}
H_{n \ell}^{(k p)}\left(\mathbf{k}_{\|}, k_{z}\right)= & \left(E_{n}(0)+\frac{\hbar^{2}\left(\mathbf{k}_{\|}^{2}+k_{z}^{2}\right)}{2 m}\right) \delta_{n \ell} \\
& +\left(\mathbf{k}_{\|}+k_{z} \hat{\mathbf{e}}_{z}\right) \cdot \mathbf{P}_{n \ell} \\
& +\sum_{\alpha \beta} \Gamma_{n \ell}^{\alpha \beta} k_{\alpha} k_{\beta}+H^{\text {(strain) }}
\end{aligned}
$$

\footnotetext{
* Corresponding author.
} 
where the indices run over the bands in our basis set $B, \mathbf{P}_{n \ell}$ are the momentum matrix elements, and $\Gamma_{n \ell}^{\alpha \beta}$ are renormalization constants describing the contribution from bands not contained in $B$. To characterize the bandstructure of crystals with zincblende symmetry near $\Gamma$, it is sufficient to include the heavy hole $(\mathrm{HH})$ and light hole bands (LH) $\left(\Gamma_{8}^{v}\right)$, the split-off bands (SO) $\left(\Gamma_{7}^{v}\right)$ and the lowest conduction bands $\left(\Gamma_{6}^{c}\right) . H^{\text {(strain) }}$, a $k$ independent term, describes the strain, which is assumed to be confined to the well region. The resulting $8 \times 8$ Hamiltonian has been described in the literature $[2,3]$.

For the superlattice calculation, $z$ is taken to be the growth direction and the usual substitution $k_{z} \rightarrow \partial_{z} / i$ is made to obtain the effective mass Eq. [4],

$$
\sum_{\ell \in \mathbf{B}} H_{m \ell}\left(\mathbf{k}_{\|}, \partial_{z} / i\right) \phi_{\ell}^{\left(n \mathbf{k}_{\|} k_{q}\right)}(z)=E^{(n)} \phi_{m}^{\left(n \mathbf{k}_{\|} k_{q}\right)}(z),
$$

where $k_{q}$ is the wave number for the 1D Brillouin zone of the superlattice, and $\phi_{m}^{\left(n \mathbf{k}_{\|} k_{q}\right)}(z)$, the superlattice envelope functions, are related to the wave functions to second order by

$$
\begin{aligned}
\psi_{n \mathbf{k} \|}= & \exp \left(i \mathbf{k}_{\|} \cdot \mathbf{r}\right) \sum_{m \in B}\left(u_{m 0}+\sum_{\ell \notin B}\right. \\
& \left.\frac{\mathbf{k}_{\|} \cdot \mathbf{P}_{\ell m}}{E_{\ell}(0)-E_{m}(0)} u_{\ell 0}\right) \phi_{m}^{\left(n \mathbf{k}_{\|} k_{q}\right)}(z) .
\end{aligned}
$$

The band parameters which enter the Hamiltonian are allowed to have different values in the well and the barrier regions [5], and the resulting operator is Fourier transformed. For a superlattice period of $500 \AA$, typically about thirty Fourier components are required to ensure convergence of the ground state eigenenergies to within $1 \mu \mathrm{eV}$. The figures throughout this paper were generated for a superlattice consisting of $\mathrm{Al}_{0.1} \mathrm{Ga}_{0.9}$ As barriers of $400 \AA$, and $\mathrm{In}_{0.2} \mathrm{Ga}_{0.8}$ As wells of $80 \AA$. The barriers are wide enough to decouple adjacent wells so that the superlattice is in practice a collection of independent quantum wells. Most band parameters were determined from [6] except for the conduction band and valence band deformation potentials used to determine band edge shifts due to strain and the fraction conduction band discontinuity, which are not well known. We assume a fraction conduction band discontinuity of 0.7 and $11 \mathrm{eV}$ for the difference in conduction band and valence band deformation potentials.

To account for carrier-carrier interactions additional terms which depend on electron and hole densities are added as diagonal terms in the k.p Hamiltonian. The direct Coulomb interaction gives the Hartree potential $V_{H}$ which is determined by solving the $1 \mathrm{D}$ Poisson equation:

$$
\partial_{z}\left(\varepsilon \partial_{z} V_{H}\right)=-\frac{4 \pi}{\varepsilon} \rho_{3 D}(z)
$$

$\rho_{3 D}(z)=\frac{\rho_{2 D}}{L} \int_{B Z} \frac{d^{2} k}{(2 \pi)^{2}} \sum_{j}\left|\phi_{j}(\mathbf{k}, z)\right|^{2} f\left(E_{j}(k), \mu\left(n_{2 D}\right)\right)$

where the envelope functions $\phi_{j}$ have been normalized to the superlattice period $L$. The Fermi distribution $f(E, \mu)$ depends on the energy dispersions found from the $\mathbf{k} \cdot \mathbf{p}$ calculation and on the quasi-chemical potential $\mu$, determined from the input parameter $n_{2 D}$, the carrier density per unit surface in one period of the superlattice.

$$
n_{2 D}=\sum_{j} \int_{B Z} \frac{d^{2} k}{(2 \pi)^{2}} f\left(E_{j}(k), \mu\right)
$$

The interaction of a carrier with its exchangecorrelation hole lowers its energy and results in a narrowing of the bandgap. This effect is taken into account in the local density approximation. We use an interpolated expression derived by Hedin and Lundquist [7] for three dimensional systems, and treat electrons and holes as separate plasmas. The bandgap renormalization (BGR) obtained is in qualitative agreement with experiment and can be improved by using an expression more appropriate for $2 \mathrm{D}$ systems.

Because these additional terms depend on the eigenfunctions determined from the $\mathbf{k} \cdot \mathbf{p}$ calcula- 
tion, an iterative approach must be used. First the k.p Hamiltonian is diagonalized for a suitable initial guess for the Hartree, exchange and correlation energies. For a given 2D carrier density, the chemical potentials are then computed assuming a Fermi distribution function. The $z$ dependence of the 3D carrier density can then be determined and used to compute a new estimate of the Hartree and exchange and correlation potentials. To assure convergence, an underrelaxation scheme is used for the exchange and correlation potentials. The approach is illustrated in the flow chart in Figure 1.

Once convergence has been attained, optical matrix elements (OME) given by $\mathbf{M}_{n n^{\prime}}=\left\langle\psi_{n k \|}\right|$ $\mathbf{p}\left|\psi_{n^{\prime} k \|}\right\rangle$ are computed for later use in MINILASE II. The square of the OME gives the strength of the electron-hole coupling through the photon interaction and therefore enters the expressions for spontaneous and stimulated emission. Figure 2a shows the OME dispersion for TE polarization and two directions of $\mathbf{k}_{\|}$, parallel to and perpendicular to $\hat{\mathbf{e}}$. At $\Gamma, \mathrm{CB} 1$ couples only to $\mathrm{HH} 1$ and

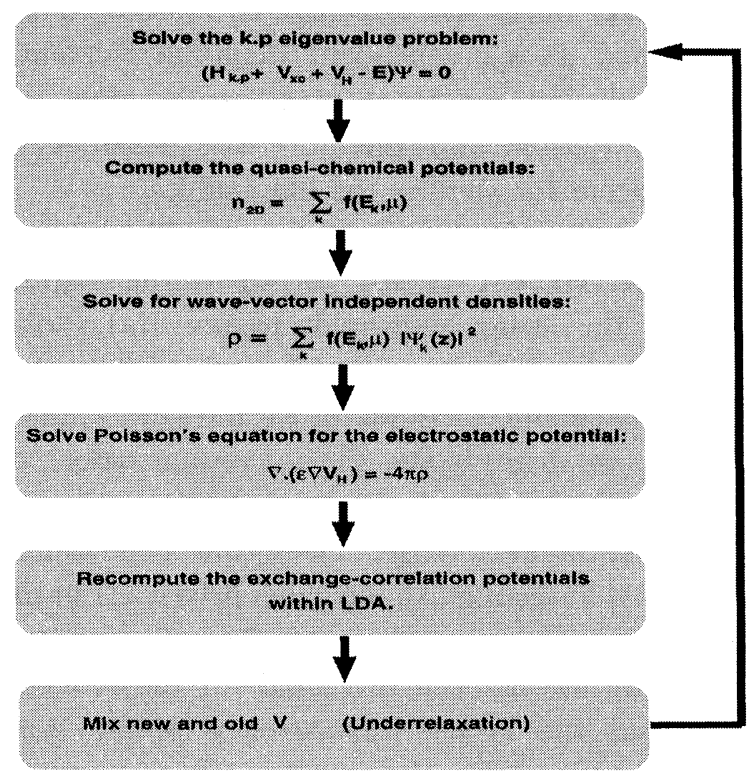

FIGURE 1 Flowchart for self-consistent $\mathbf{k} \cdot \mathbf{p}$ calculation.
LH1 because of the orthogonality relation between the envelope functions. However, away from $\Gamma$, band mixing allows formerly forbidden transitions to take place. As expected for this polarization, the coupling at $\Gamma$ between $\mathrm{CB} 1$ and $\mathrm{HH} 1$ is about three times stronger than between $\mathrm{CB} 1$ and $\mathrm{LH} 1$ [8].

Due to the Coulomb attraction between electrons and holes, spontaneous and stimulated emission are enhanced. This Coulomb enhancement, which may be thought of a renormalization of the optical matrix elements, is computed external to MINILASE II for various carrier densities and lasing energies. The expression used for the Coulomb enhancement factor has been
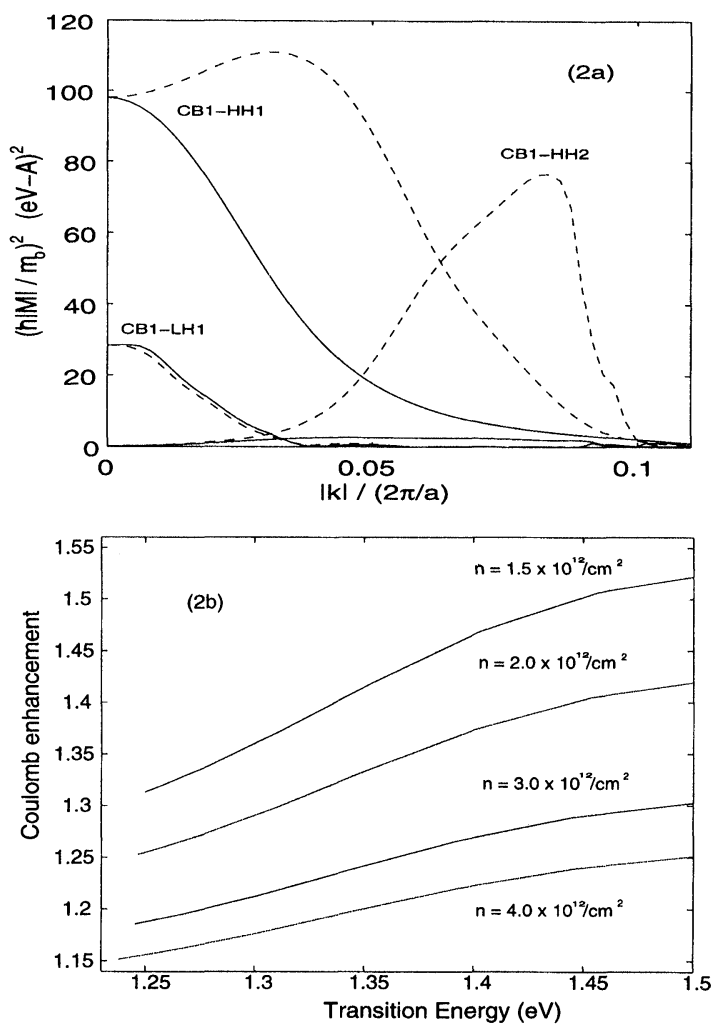

FIGURE 2 (a) Optical matrix elements for $\mathbf{k}_{\|}$parallel to (solid) and normal to (dashed) the polarization. Transitions between the lowest conduction subband $\mathrm{CB} 1$ and the valence subbands HH1, HH2 and LH1 are shown. (b) Density dependence of the Coulomb enhancement factor. 
derived in the literature [9] and is given by

$$
\frac{1}{1-q(k)}
$$

where

$$
\begin{aligned}
q(k)= & \frac{1}{M(k)} \int d k^{\prime} V^{(s)}\left(k-k^{\prime}\right) \\
& \frac{i M\left(k^{\prime}\right)}{\hbar} \frac{f_{n}\left(k^{\prime}\right)+f_{p}\left(k^{\prime}\right)-1}{i\left(E_{c}\left(k^{\prime}\right)-E_{v}\left(k^{\prime}\right)-\nu\right)+1 / \tau}
\end{aligned}
$$

Here $f_{n}$ and $f_{p}$ are Fermi occupation factors, $\tau$ is the carrier-carrier scattering time, assumed to be $100 \mathrm{fs}$, and $V^{(s)}$ is the 2D screened Coulomb potential which in the static long wavelength limit of RPA is given by

$$
V^{(s)}(q)=\frac{2 \pi e^{2}}{\varepsilon_{0} q} \frac{1}{1+\kappa / q}
$$

where

$$
\kappa=\frac{2 \pi e^{2}}{\varepsilon_{0}}\left(\frac{\partial n}{\partial \mu_{n}}+\frac{\partial p}{\partial \mu_{p}}\right)
$$

is the inverse screening length. Figure $2 b$ shows the renormalization factor for the $\mathrm{CB} 1-\mathrm{HH} 1$ transition between the lowest conduction subband and lowest valence subband for several carrier densities above threshold. In this calculation, an axial average of the matrix elements is performed, so that the OME may be treated strictly as a function of energy rather than in-plane wave vector $\mathbf{k}_{\|}$. We see that the modest enhancement of the OME decreases as the carrier density increases. This result stems from the fact that screening is enhanced at higher densities.

The results of these calculations are exported to MINILASE II in three forms: the spatial profile of the envelope functions, a density of states and an optical matrix element factor $P_{n}(\Omega)$ (associated with each conduction band energy grid point $n$ ) given by

$$
\begin{aligned}
P_{n}(\Omega)= & \sum_{i j} \int \frac{d^{2} k_{\|}}{(2 \pi)^{2}}\left|\hat{\mathbf{e}} \cdot \mathbf{M}_{i j}\left(\mathbf{k}_{\|}\right)\right|^{2} \eta_{n}\left(E_{c}^{i}\left(\mathbf{k}_{\|}\right)\right) \\
& \delta\left(E_{c}^{i}\left(\mathbf{k}_{\|}\right)-E_{v}^{j}\left(\mathbf{k}_{\|}\right)-\Omega\right)
\end{aligned}
$$

The direction of the light polarization is $\hat{\mathbf{e}}$, and $\eta_{n}$ is a hat function with support on the energy range defined by grid points $n-1$ and $n+1$ and whose area is normalized to unity. The sum extends over all conduction subbands $i$ and valence subbands $j$. The gain coefficient is then given by

$$
\begin{aligned}
g^{(0)}(\Omega) \sim & \frac{1}{\hbar \Omega} \sum_{n}\left(\tilde{f}_{e}\left(\varepsilon_{n}\right)+\tilde{f}_{h}\left(\varepsilon_{n}-\Omega\right)-1\right) \\
& P_{n}(\Omega) \mathcal{L}(\Omega, \tau)
\end{aligned}
$$

where $\mathcal{L}$ is a Lorentzian broadening function and $\tilde{f}_{e}$ and $\tilde{f}_{h}$ are the non-equilibrium electron and hole distribution functions, respectively. Minilase II incorporates the k.p data in an iterative manner. That is, the simulator first solves the carrier transport equations, Poisson's equation and the photon rate equations through Newton's method. The resulting carrier densities are used to recompute the band edges from an interpolated expression for the bandgap renormalization. Then, files appropriate for the computed density are read from disk. Since the exchange-correlation terms are added as k-independent diagonal elements, their inclusion does not significantly alter the effective masses and therefore the densities of state, except for shifting the band-edges.

We now briefly describe a few results obtained from MINILASE-II for an operating regime beyond the lasing threshold. Figure 3(a) shows the optical gain spectra with and without the Coulomb enhancement factor for the same applied current. In steady state, the maximum height of the gain is pinned by the losses in the laser which is the same in each case. There are two salient features that distinguish the gain curve without the Coulomb enhancement from the other curve. These are the reduced threshold for the onset of gain and the larger transparency point. Both features stem from the smaller effective matrix element in the absence of Coulomb enhancement 

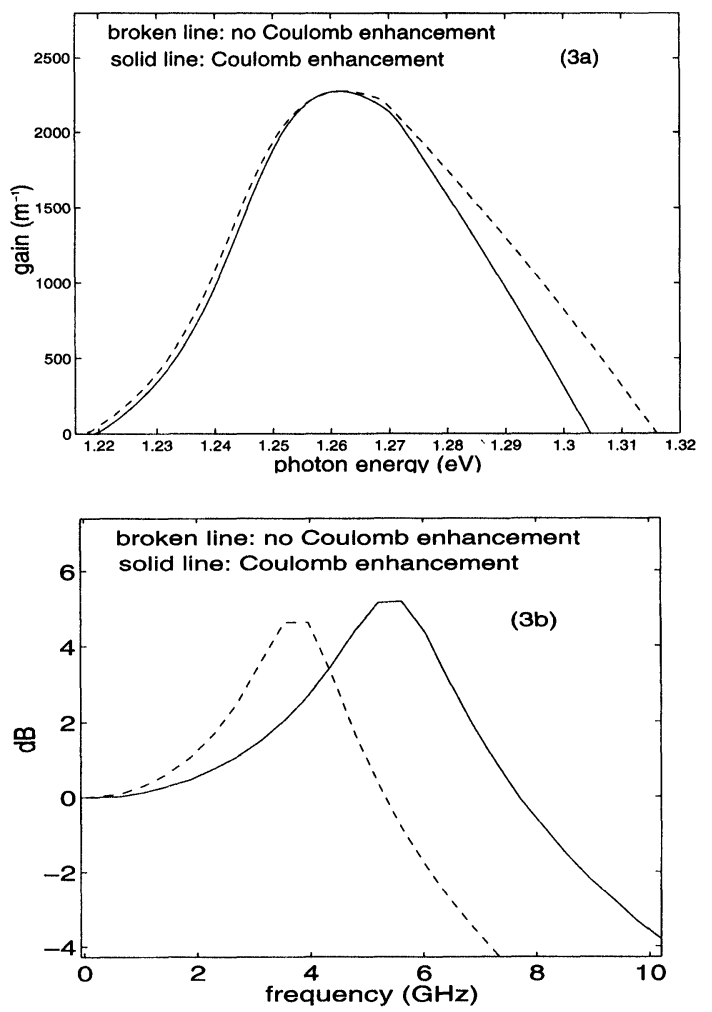

FIGURE 3 Gain with and without Coulomb enhancement.

that requires greater pumping of the laser to achieve a fixed gain. Thus, more carriers are required which results in a reduction in the band gap and implies a greater separation of the electron and hole quasi-fermilevels and thus a larger transparency point.

Figure 3(b) displays the laser's response to a small square pulse in the applied voltage. We note that the frequency peak is blue shifted when a larger effective matrix element is used. This may be explained as follows. The photon rate equation has the form

$$
\dot{S}_{\nu}=\left(G_{\nu}-1 / \tau_{\gamma}\right) S_{\nu}+R
$$

where $\nu$ is a mode index, $S_{\nu}$ is the photon population, $G_{\nu}$ is the modal gain, $\tau_{\gamma}$ is the photon lifetime and $R$ is the spontaneous emission rate, which is much smaller than the other terms of the right hand side under lasing conditions. Since the losses $1 / \tau$ are fixed, the gain is roughly proportional to the time derivative of the photon density in the cavity which implies a more swiftly responding laser.

In conclusion, we described how an accurate $\mathbf{k} \cdot \mathbf{p}$ calculation was connected to a sophisticated laser simulator and demonstrated how the larger effective matrix element that stems from the Coulomb enhancement affects the gain and resonance frequency in the modulation response of a laser.

\section{Acknowledgement}

We acknowledge the support of NSF through NCCE and of the Office of Naval Research.

\section{References}

[1] Grupen, M., Hess, K. and Rota, L. "Simulating Spectral Hole Burning and the Modulation Response of Quantum Well Laser Diodes", Proc. of the SPIE Conf., 6-9 February 1995, San Jose, California.

[2] Cardona, M., Christensen, N. E. and Fasol, G. "Relativistic Band Structure and Spin Orbit Splitting of Zinc-blendetype Semiconductors", Phys. Rev. B, 38, 1806, July 15, 1988.

[3] O'Reilly, E. P. (1989). "Valence Band Engineering in Strained-Layer Structures", Semicond. Sci. Technol., 4, 121.

[4] von Allmen, P. (1992). "Derivation of the Effective Mass Equation for a Superlattice: a Perturbational Approach", Phys. Rev. B, 46, 15377, December 15.

[5] von Allmen, P. "Conduction Subbands in a GaAs/ $\mathrm{Al}_{x} \mathrm{Ga}_{1-x} \mathrm{As}$ Quantum Well: Comparing Different k·p models", Phys. Rev. B, 46, 15382, December 15, 1992.

[6] Landoldt-Bornstein, L. (1982). "Numerical Data and Functions in Science and Technology", edited by $O$. Madelung. Berlin: Springer, 17a and 22a.

[7] Hedin, L. and Lundquist, B. I. (1971). "Explicit local exchange correlation potentials", J. Phys. C, 4, 2064.

[8] Chuang, S. L. (1995). Physics of Optoelectronic Devices, John Wiley and Sons.

[9] Weng Chow, Stephen Koch and Murray Sargent. (1994). Semiconductor Laser Physics, Springer-Verlag.

\section{Authors' Biographies}

Fabiano Oyafuso received B.A. degrees in mathematics (with honors) and physics from UC Berkeley in 1992. He is currently pursuing the $\mathrm{Ph} . \mathrm{D}$. degree in physics at the University of Illinois 
at Urbana-Champaign. His thesis involves the study of the temperature dependence of threshold current densities in quantum well lasers.

Paul von Allmen obtained his B.S. and Ph.D. in physics from the Swiss Federal Institute of Technology in Lausanne. He joined the Zurich IBM research laboratory as a postdoctoral research associate in 1990. Since 1992 he has been an invited scholar at the Beckman Institute for Advanced Science and Technology at the University of Illinois in Urbana-Champaign. His interests range from subband structures and many-body effects in confined electron systems to the dynamical properties of nanostructures and surfaces. His main field of interest is presently the study of the desorption mechanism of hydrogen and deuterium from a silicon surface and the related isotope effect which has important consequences for the resistance of MOS-transistors against hot carrier degradation.

Matt Grupen received his B.S. from Penn State University in Engineering Science n 1985. He then attended UCLA where he received an M.S. in Electron Device Physics in 1989. In 1994, he received a Ph.D. in Computational Electronics at the University of Illinois, where he has held a postdoctoral position.

Karl Hess has dedicated the major portion of his research career to the understanding of electronic current flow in semiconductors and semiconductor devices with particular emphasis on effects pertinent to device miniaturization. His theories and use of large computer resources are aimed at complex problems with clear applications and relevance to miniaturization of electronics. $\mathrm{He}$ is currently the Swanlund Professor of Electrical and Computer Engineering, Professor of Physics, Adjunct Professor for Supercomputing Applications and a Research Professor in the Beckman Institute working on topics related to Molecular and Electronic Nanostructures. He has received numerous awards, for example the IEEE David Sarnoff Field Award for electronics in 1995. 

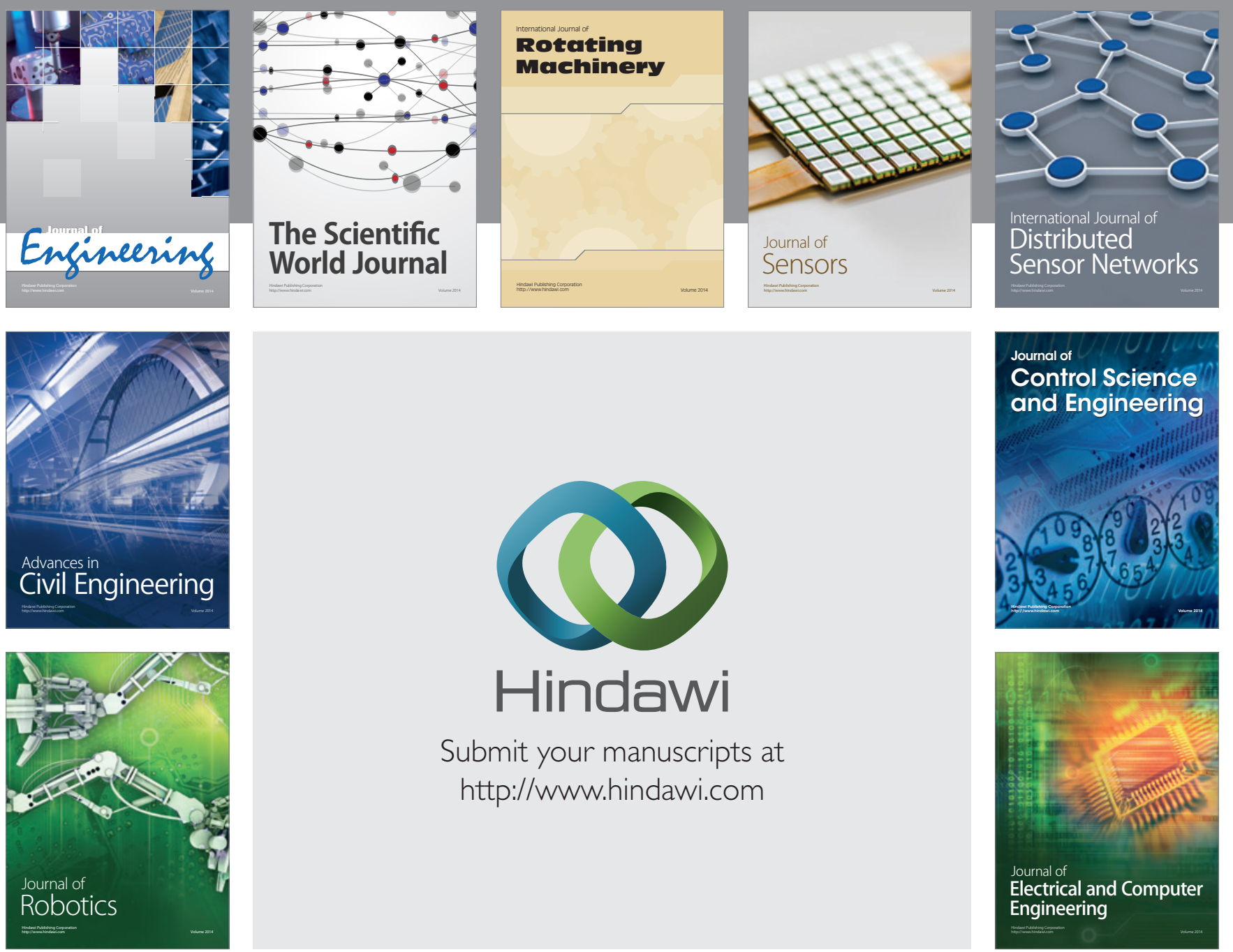

Submit your manuscripts at

http://www.hindawi.com
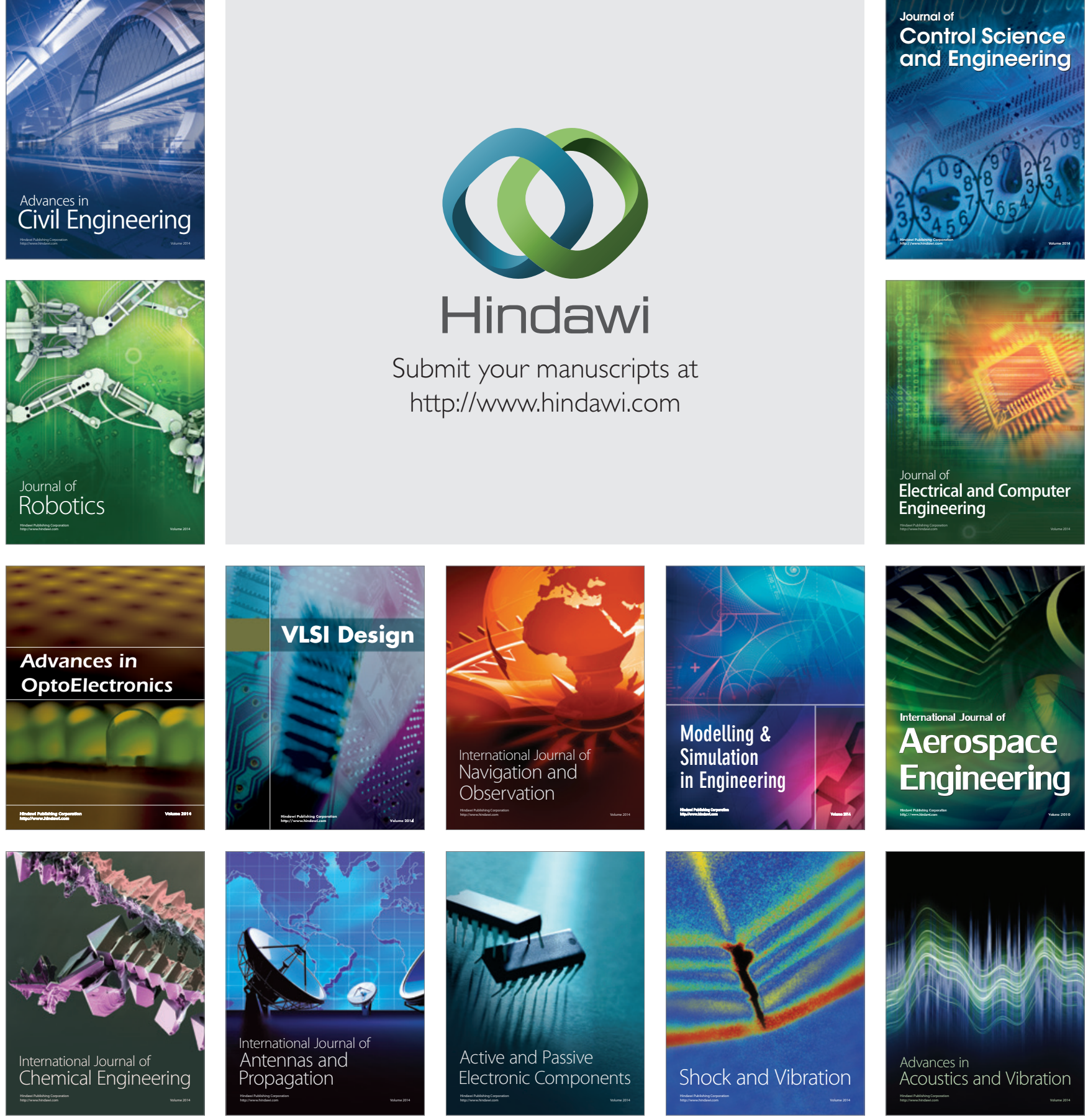